\title{
A multivariable prognostic score to guide systemic therapy in early-stage HER2-positive breast cancer: a retrospective study with an external evaluation
}

\author{
Aleix Prat, Valentina Guarneri, Laia Paré, Gaia Griguolo, Tomás Pascual, Maria V Dieci, Núria Chic, Blanca González-Farré, Antonio Frassoldati, \\ Esther Sanfeliu, Juan M Cejalvo, Montserrat Muñoz, Giancarlo Bisagni, Fara Brasó-Maristany, Loredana Urso, Maria Vidal, Alba A Brandes, \\ Barbara Adamo, Antonino Musolino, Federica Miglietta, Benedetta Conte, Mafalda Oliveira, Cristina Saura, Sònia Pernas, Jesús Alarcón, \\ Antonio Llombart-Cussac, Javier Cortés, Luis Manso, Rafael López, Eva Ciruelos, Francesco Schettini, Patricia Villagrasa, Lisa A Carey, \\ Charles M Perou, Federico Piacentini, Roberto D’Amico, Enrico Tagliafico, Joel S Parker, Pierfranco Conte
}

\section{Summary}

Background In early-stage HER2-positive breast cancer, escalation or de-escalation of systemic therapy is a controversial topic. As an aid to treatment decisions, we aimed to develop a prognostic assay that integrates multiple data types for predicting survival outcome in patients with newly diagnosed HER2-positive breast cancer.

Methods We derived a combined prognostic model using retrospective clinical-pathological data on stromal tumourinfiltrating lymphocytes, PAM50 subtypes, and expression of 55 genes obtained from patients who participated in the Short-HER phase 3 trial. The trial enrolled patients with newly diagnosed, node-positive, HER2-positive breast cancer or, if node negative, with at least one risk factor (ie, tumour size $>2 \mathrm{~cm}$, histological grade 3 , lymphovascular invasion Ki67 $>20 \%$, age $\leq 35$ years, or hormone receptor negativity), and randomly assigned them to adjuvant anthracycline plus taxane-based combinations with either 9 weeks or 1 year of trastuzumab. Trastuzumab was administered intravenously every 3 weeks $(8 \mathrm{mg} / \mathrm{kg}$ loading dose at first c ycle, a nd $6 \mathrm{mg} / \mathrm{kg}$ thereafter) for $18 \mathrm{~d}$ oses or w eekly $(4 \mathrm{mg} / \mathrm{kg}$ loading dose in the first w eek, and $2 \mathrm{mg} / \mathrm{kg}$ thereafter) for 9 w eeks, $\mathrm{s}$ tarting concomitantly with the firs taxane dose. Median follow-up was 91.4 months (IQR 75·1-105.6). The primary objective of our study was to derive and evaluate a combined prognostic score associated with distant metastasis-free survival (the time between randomisation and distant recurrence or death before recurrence), an exploratory endpoint in Short-HER. Patient samples in the training dataset were split into a training set $(n=290)$ and a testing set $(n=145)$, balancing for event and treatment group. The training set was further stratified into 100 iterations of Monte-Carlo cross validation (MCCV). Cox proportional hazard models were fit to MCCV training samples using Elastic-Net. A maximum of 92 features were assessed. The final prognostic model was evaluated in an independent combined dataset of 267 patients with earlystage HER2-positive breast cancer treated with different neoadjuvant and adjuvant anti-HER2-based combinations and from four other studies (PAMELA, CHER-LOB, Hospital Clinic, and Padova) with disease-free survival outcome data.

Findings From Short-HER, data from 435 (35\%) of 1254 patients for tumour size (T1 vs rest), nodal status (N0 vs rest), number of tumour-infiltrating lymphocytes (continuous variable), subtype (HER2-enriched and basallike vs rest), and 13 genes composed the final model (named HER2DX). HER2DX was significantly associated with distant metastasis-free survival as a continuous variable $(\mathrm{p}<0.0001)$. HER2DX median score for quartiles $1-2$ was identified as the cutoff to identify low-risk patients; and the score that distinguished quartile 3 from quartile 4 was the cutoff to distinguish medium-risk and high-risk populations. The 5-year distant metastasis-free survival of the

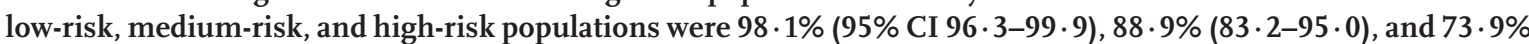
$(66 \cdot 0-82 \cdot 7)$, respectively (low-risk vs high-risk hazard ratio [HR] $0 \cdot 04,95 \%$ CI $0 \cdot 0-0 \cdot 1, \mathrm{p}<0 \cdot 0001)$. In the evaluation cohort, HER2DX was significantly a ssociated $\mathrm{w}$ ith $\mathrm{d}$ isease-free $\mathrm{s}$ urvival a $\mathrm{s}$ a $\mathrm{c}$ ontinuous $\mathrm{v}$ ariable (HR 2.77, 95\% CI 1·4-5 -6, p=0 0040) and as group categories (low-risk vs high-risk HR 0 27, 0 1-0 7, p=0 005). 5-year disease-free survival in the HER2DX low-risk group was $93 \cdot 5 \%(89 \cdot 0-98 \cdot 3 \%)$ and in the high-risk group was $81 \cdot 1 \%(71 \cdot 5-92 \cdot 1)$.

Interpretation The HER2DX combined prognostic score identifies patients with early-stage, HER2-positive breast cancer who might be candidates for escalated or de-escalated systemic treatment. Future clinical validation of HER2DX seems warranted to establish its use in different scenarios, especially in the neoadjuvant setting.

Funding Instituto Salud Carlos III, Save the Mama, Pas a Pas, Fundación Científica, Asociación Española Contra el Cáncer, Fundación SEOM, National Institutes of Health, Agenzia Italiana del Farmaco, International Agency for Research on Cancer, and the Veneto Institute of Oncology, and Italian Association for Cancer Research.

Lancet Oncol 2020; 21: 1455-64 See Comment page 1392 SOLTI Breast Cancer Research Group, Barcelona, Spain (Prof A Prat MD, L Paré PhD, T Pascual MD, N Chic MD, B González-Farré MD, E Sanfeliu MD, M Muñoz MD F Brasó-Maristany PhD, M Vidal MD, B Adamo MD M Oliveira MD, C Saura MD SPernas MD, J Alarcón MD, L Manso MD, R López MD, E Ciruelos MD, F Schettini MD, P Villagrasa PhD); Department of Medical Oncology, Hospital Clinic of Barcelona, Barcelona Spain (Prof A Prat, N Chic, M Muñoz, M Vidal, B Adamo); Translational Genomics and Targeted Therapeutics in Solid Tumors, August Pi i Sunyer Biomedical Research Institute (IDIBAPS), Barcelona, Spain (Prof A Prat, T Pascual, N Chic, B González-Farré, E Sanfeliu, M Muñoz, F Brasó-Maristany, M Vidal, B Adamo, B Conte MD, FSchettini); Department of Medicine, University of Barcelona, Barcelona, Spain (Prof A Prat); Department of Surgery, Oncology and Gastroenterology, University of Padova, Padova, Italy (V Guarneri MD, G Griguolo MD $M V$ Dieci MD, F Miglietta MD Prof P Conte MD); Medical Oncology 2, Istituto Oncologico Veneto, IRCCS, Padova, Italy (V Guarneri, G Griguolo, M V Dieci, L Urso MD, F Miglietta, Prof P Conte); Lineberger Comprehensive Cancer Center, Department of Genetics, University of North Carolina at Chapel Hill, Chapel Hill, NC, USA (T Pascual, LA Carey MD, C M Perou PhD, J S Parker PhD); Department of Pathology, Hospital Clinic of Barcelona, Barcelona, Spain (B González-Farré, E Sanfeliu); Clinical Oncology, Department 
of Morphology, Surgery and Experimental Medicine, S Anna University Hospital, Ferrara, Italy (A Frassoldati MD); Department of Medical Oncology, Hospital Clínico Universitario of Valencia, Valencia, Spain

(J M Cejalvo MD); Pathology Unit, Azienda Unità Sanitaria Locale-IRCCS di Reggio Emilia, Reggio Emilia, Emilia-Romagna, Italy (G Bisagni MD); Medical Oncology, Azienda Unità Sanitaria Locale di BolognaIRCCS Istituto delle Scienze Neurologiche, Bologna (A A Brandes MD); Department of Medicine and Surgery, and the Medical Oncology and Breast Unit, University Hospital of Parma, Piacenza, Italy (A Musolino MD); Department of Medical Oncology UO Oncologia Medica 2, IRCCS Ospedale Policlinico San Martino, Genova, Italy (B Conte): Department of Medical Oncology, Vall d'Hebron University Hospital; Vall d'Hebron Institute of Oncology, Barcelona, Spain (M Oliveira, C Saura,

J Cortés MD); Department of Medical Oncology, Institut Català d'Oncologia Hospitalet, Hospitalet de Llobregat, Spain (S Pernas); Hospital Universitario Son Espases, Carretera de Valldemossa, Palma de Mallorca, Spain (J Alarcón); Department of Medical Oncology, Hospital Arnau de Vilanova, Valencia, Spain (A Llombart-Cussac MD); IOB Institute of Oncology, Quiron Group, Barcelona, Spain (J Cortés); Department of

Medical Oncology, Hospital

12 de Octubre, Madrid, Spain (L Manso, E Ciruelos); Department of Medical Oncology, Complejo

Universitario de Santiago de Compostela-CIBERONC, Santiago de Compostela, Spain

(R López); Department of Medical Oncology, University of Naples Federico II, Naples, Italy (F Schettini);

and Department of Medical and Surgical Sciences (F Piacentini MD, R D'Amico MD, E Tagliafico MD) and Center for Genome Research, University of Modena and Reggio Emilia,

Modena, Italy (R D'Amico, ETagliafico)

\section{Research in context}

\section{Evidence before this study}

We searched PubMed for clinical trials or studies published in English between Jan 1, 2010, and May 1, 2020, assessing HER2 inhibition in early-stage breast cancer, with the search terms "HER2+", "early-stage", "escalation", "de-escalation", "biomarker", "breast cancer", and "anti-HER2 therapy". To date, several variables associated with survival outcome have been identified in early-stage, HER2-positive breast cancer, such as TNM staging before and after neoadjuvant therapy, hormone receptor status, tumour-infiltrating lymphocytes, PAM50 intrinsic subtype, and PIK3CA mutations. However, validation and clinical utility of these biomarkers, either alone or in combination, remains unknown.

International guidelines support the administration of adjuvant or neoadjuvant anti-HER2-based chemotherapy in patients with T1b-T4 or lymph-node positive disease. Since 2010 however, various strategies to either escalate or de-escalate systemic therapy in early-stage, HER2-positive breast cancer have been evaluated, such as (1) decreasing the amount of chemotherapy, (2) decreasing the duration of trastuzumab, (3) increasing HER2 blockade with either the addition of 1 year of pertuzumab to trastuzumab or the addition of 1 year of neratinib after trastuzumab, and (4) switching the type of anti-HER2 therapy to trastuzumab emtansine in patients who do not achieve a pathological complete response following neoadjuvant trastuzumab-based chemotherapy. Despite the successes and limitations of these treatment strategies, most patients with early-stage, HER2-positive breast cancer are cured with chemotherapy and

\section{Introduction}

HER2-positive breast cancer is responsible for a substantial proportion of deaths in women. ${ }^{1}$ In the early stages, adjuvant or neoadjuvant chemotherapy and anti-HER2 therapy (plus endocrine therapy in hormone receptor-positive disease) have consistently shown significant and long-term clinical benefits, in terms of disease-free survival and overall survival. ${ }^{1}$ However, substantial heterogeneity exists in HER2-positive disease regarding tumour biology, ${ }^{2-6}$ patients' prognosis, ${ }^{7}$ and treatment benefit.

Strategies to either escalate or de-escalate systemic therapy in early-stage HER2-positive breast cancer to improve survival outcomes have been explored, ${ }^{8}$ such as decreasing the number of cycles of chemotherapy ${ }^{9}$ and the duration of trastuzumab, ${ }^{10}$ increasing HER2 blockade with pertuzumab ${ }^{11}$ or neratinib,$^{12}$ or switching anti-HER2 therapy to trastuzumab emtansine in patients who did not achieve a pathological complete response following neoadjuvant trastuzumab-based chemotherapy. ${ }^{13}$ Despite these changes, most patients with early-stage, HER2positive breast cancer are cured with chemotherapy and trastuzumab. ${ }^{14}$ trastuzumab; therefore, a multivariable prognostic tool to help guide systemic therapies in early-stage, HER2-positive breast cancer is needed.

\section{Added value of this study}

To our knowledge, ours is the first study attempting to build a combined prognostic score (called HER2DX) based on

17 clinicopathological and genomic variables in early-stage, HER2-positive breast cancer, using tumour samples from a phase 3 clinical trial. Additionally, the prognostic score was evaluated in a combined neoadjuvant dataset of patients with newly diagnosed, HER2-positive breast cancer who received anti-HER2-based therapy, providing insights about the relationship between response to therapy in the neoadjuvant setting and long-term survival outcome.

\section{Implications of all the available evidence}

The evidence suggests that the HER2DX prognostic score identified a substantial proportion of patients with early-stage, HER2-positive breast cancer who might not need additional therapies, such as pertuzumab, neratinib, or trastuzumab emtansine because of their favourable survival outcomes with chemotherapy and trastuzumab (plus endocrine therapy if hormone receptor-positive). Further studies should establish the clinical use of the HER2DX prognostic score in this context and explore its value to help further de-escalate systemic treatments, such as the duration of trastuzumab or the amount of chemotherapy. Finally, multivariable prognostic models should be explored in other breast cancer subtypes, such as triple-negative disease, and other cancer types.

In early-stage hormone receptor-positive and HER2negative breast cancer, several prognostic tools allow better individualisation of systemic treatments and are widely available. For example, gene expression-based assays such as OncotypeDX (Genomic Health, Redwood City, CA, USA $)^{15}$ help to identify low-risk patients who do not need adjuvant or neoadjuvant chemotherapy. Second-generation genomic tests, such as PAM50/Prosigna (NanoString Technologies, Seattle, WA, USA) ${ }^{16}$ which include clinical variables such as tumour size in the final risk assessment, might better distinguish patients who might not need chemotherapy from those who are likely to benefit.

Some variables beyond the tumour-node-metastasis classification have been associated with prognosis in early-stage, HER2-positive breast cancer-eg, staging before and after neoadjuvant therapy, hormone receptor status, number of stromal tumour-infiltrating lymphocytes, ${ }^{14,17,18}$ and PAM50 intrinsic subtypes. ${ }^{2,18,19}$ Similarly, these biomarkers and PIK3CA mutations ${ }^{20}$ have been associated with the probability of achieving a pathological complete response, ${ }^{20,21}$ which is also associated with a positive long-term outcome. ${ }^{22}$ However, decisions about escalation or de-escalation of systemic therapies are 
based on nodal status, hormone receptor status, and therapy response..$^{23}$ Therefore, a multivariable prognostic tool that integrates several variables to help guide systemic therapies in early-stage, HER2-positive breast cancer is urgently needed. Here, we aimed to develop such a prognostic tool based on multiple variables.

\section{Methods \\ Study design and participants}

This combined prognostic model was derived using retrospective clinical, pathological, and genomic data from a subset of patients who participated in the Short-HER trial. The final prognostic model was evaluated retrospectively in a combined and independent cohort of patients from four other studies (CHER-LOB, PAMELA, Hospital Clinic, and Padova) with early-stage, HER2-positive breast cancer.

Short-HER was a randomised, investigator-driven phase 3 study that aimed to assess the non-inferiority of 9-week versus 1-year adjuvant trastuzumab combined with chemotherapy in terms of disease-free survival (primary endpoint) in women with HER2-positive breast cancer. ${ }^{24}$ Briefly, eligible participants were women aged 18-75 years with surgically resected, HER2-positive breast cancer with node positivity, or in case of node negativity, at least one of the following features: tumour size larger than $2 \mathrm{~cm}$, histological grade 3 , presence of lymphovascular invasion, Ki67greater than $20 \%$, age 35 years or younger, or hormone receptor negativity. 1254 patients with an Eastern Cooperative Oncology Group (ECOG) performance status of $0-1$ were randomly assigned from Dec 17, 2007, to Oct 6, 2013, to one of two treatment groups. The first group was assigned chemotherapy consisting of intravenous doxorubicin $60 \mathrm{mg} / \mathrm{m}^{2}$ or intravenous epirubicin $90 \mathrm{mg} / \mathrm{m}^{2}$ plus intravenous cyclophosphamide $600 \mathrm{mg} / \mathrm{m}^{2}$ every 3 weeks for four courses followed by intravenous paclitaxel $175 \mathrm{mg} / \mathrm{m}^{2}$ or docetaxel $100 \mathrm{mg} / \mathrm{m}^{2}$ every 3 weeks for four courses. Trastuzumab was administered intravenously every 3 weeks $(8 \mathrm{mg} / \mathrm{kg}$ loading dose at first cycle, and $6 \mathrm{mg} / \mathrm{kg}$ thereafter) for 18 doses, starting with the first taxane dose. The second group was assigned chemotherapy consisting of intravenous docetaxel $100 \mathrm{mg} / \mathrm{m}^{2}$ every 3 weeks for three courses followed by intravenous fluorouracil $600 \mathrm{mg} / \mathrm{m}^{2}$, epirubicin $60 \mathrm{mg} / \mathrm{m}^{2}$, and cyclophosphamide $600 \mathrm{mg} / \mathrm{m}^{2}$ every 3 weeks for three courses. Trastuzumab was administered weekly $(4 \mathrm{mg} / \mathrm{kg}$ loading dose in the first week, and $2 \mathrm{mg} / \mathrm{kg}$ thereafter) for 9 weeks, starting concomitantly with docetaxel. When indicated, radiotherapy and hormonal therapy were given according to local standard care. Median follow-up was 91.4 months (IQR 75·1-105·6), and distant metastasis-free survival (the time between randomisation and distant recurrence or death before recurrence) was an exploratory endpoint.

CHER-LOB ${ }^{25}$ was a randomised, non-comparative, investigator-driven phase 2 study done from Aug 8, 2006, to Nov 25, 2010, of preoperative taxane anthracycline consisting of intravenous paclitaxel $\left(80 \mathrm{mg} / \mathrm{m}^{2}\right)$ for 12 weeks followed by intravenous fluorouracil, epirubicin, and cyclophosphamide for four courses every 3 weeks, in combination with intravenous trastuzumab, intravenous trastuzumab plus $1000 \mathrm{mg}$ oral lapatinib (daily) or $1500 \mathrm{mg}$ lapatinib (daily) for 26 weeks in patients with HER2-positive, stage II to IIIA operable breast cancer and with a performance status of ECOG $0-1$. The primary endpoint was to estimate the pathological complete response rate. Treatment after surgery was left to treating physician discretion. Median follow-up was 60.0 months (IQR 46.9-69.4), and disease-free survival (the time between treatment initiation and any of the following events, whichever occurred first: local, regional, and distant recurrence; contralateral breast cancer, other second invasive primary cancer, death before recurrence, or second primary cancer) was an exploratory endpoint.

PAMELA was a single-group, phase 2 trial done from Oct 22, 2013, to Nov 30, 2015, which aimed to assess the ability of the PAM50 HER2-enriched subtype to predict pathological complete response (the primary endpoint) at the time of surgery. ${ }^{21}$ Patients with HER2-positive breast cancer, stage I-IIIA, and an ECOG performance status of $0-1$ were given oral lapatinib (1000 mg per day) and intravenous trastuzumab for 18 weeks; hormone receptor-positive patients were additionally given oral letrozole ( $2.5 \mathrm{mg}$ per day) or oral tamoxifen $(20 \mathrm{mg}$ per day) according to menopausal status. Treatment after surgery was left to the treating physician's discretion. Median follow-up was 68.1 months (IQR 57.1-72.3), and disease-free survival was an exploratory endpoint.

The Hospital Clinic and Padova University cohorts are consecutive series of patients with early-stage, HER2positive breast cancer and an ECOG performance status of $0-1$ treated as per standard practice from June 28,2005 , to Sept 26, 2018 (Hospital Clinic), and Feb 23, 2009, to May 26, 2016 (Padova University cohort), with neoadjuvant trastuzumab-based chemotherapy for 3-6 months, followed by surgery. Adjuvant treatment was completed with trastuzumab for up to 1 year. When indicated, radiotherapy and hormonal therapy were carried out according to local standard care. Median follow-up of the Hospital Clinic and Padova University cohorts were 39.3 (IQR 29.6-55.8) and 38.5 (IQR 30.1-65.7) months, respectively. In both cohorts, disease-free survival was an exploratory endpoint. Approvals for the original studies were obtained from independent ethics committees.

\section{Procedures}

PAM50 and single gene analyses were done at the August Pi i Sunyer Biomedical Research Institute using formalin-fixed, paraffin-embedded tumours. Samples analysed from Short-HER were from surgical specimens, whereas samples analysed from the neoadjuvant cohorts from the other studies were from baseline samples before starting neoadjuvant therapy. A minimum of around $125 \mathrm{ng}$ of total RNA was used to measure the
Correspondence to: Prof Aleix Prat, Hospital Clínic of Barcelona, Translational Genomics and Targeted Therapies in Solid Tumors, August Pi i Sunyer Biomedica Research Institute (IDIBAPS) Barcelona, 08035, Spain alprat@clinic.cat@prat_aleix 
expression of the 50 PAM50 subtype predictor genes and five other genes (CD8A, PD-L1, PD-1,CD4, and $A R$ ). Normalisation using housekeeping genes, and PAM50 subtyping with adjustment factors, were done as previously described. ${ }^{21}$ For samples from CHER-LOB, PAM50 gene expression and subtyping values were obtained from PAM50-based microarray data (using the Affymetrix Human Genome U133 Plus 2.0 array) as previously described. ${ }^{26}$ All gene expression analyses were done blinded from clinical data. Nodal and tumour stages were obtained from clinical report forms. Finally, tumour-infiltrating lymphocytes, which consisted of all lymphocytic cell populations that had invaded the tumour tissue were assessed according to predefined criteria for the formalin-fixed paraffin-embedded tumour samples from all studies. ${ }^{27}$

\section{Outcomes}

The primary objective of this study was to derive and evaluate a combined prognostic score, named HER2DX, as a continuous variable. In the training dataset (ie, data from Short-HER), the chosen survival endpoint was distant metastasis-free survival, similar to studies of other gene expression-based prognostic biomarkers such as PAM50 risk of recurrence in hormone receptorpositive, HER2-negative breast cancer. ${ }^{16}$ In the evaluation dataset, the survival endpoint was disease-free survival because of the availability of the data.

Our secondary objectives in the training and testing sets were (1) to describe the clinical-pathological and genomic features of the HER2DX risk groups; (2) to explore the association of HER2DX score with diseasefree survival in the evaluation dataset according to the type of pathological response; (3) to evaluate the association of HER2DX score with pathological complete response in the breast and axilla in the evaluation dataset. We also did an analysis of the association of HER2DX with disease-free survival in Short-HER.

\section{Statistical analysis}

For description purposes, 5-year estimates of distant metastasis-free survival, and 5-year and 8-year diseasefree survival estimates were calculated by Kaplan-Meier. The prognostic model was developed using a training dataset of patients enrolled in the Short-HER trial (appendix p 1). The rule to define a patient assessable in Short-HER was availability of gene expression, clinicalpathological, and tumour-infiltrating lymphocytes data. Patients were divided into a training set and a testing set, balancing for distant metastasis-free survival events and treatment group. The training set was further stratified into 100 iterations of Monte-Carlo cross validation (MCCV). Cox proportional hazard models were fitted to MCCV training cases using elastic-net (package glmnet). A maximum of 92 features were evaluated. Elastic-net parameters ( $\alpha$ and $\lambda$ ) were selected to reduce the partial likelihood of deviance and increase Harrell's C-index evaluated in the MCCV test sets. These selected values were then used to fit our final model against the complete training set. A total of 17 variables were selected with the following survival coefficients: nodal stage N1-3 (0-680), tumour size T2-4 (0.339), MMP11 $(0 \cdot 200)$, PAM50 HER2-enriched or basal-like $(0.156)$, CDC6 $(0.087)$, CDH3 (0.076), TMEM45B (0.048), EXO1 (0.024), FGFR4 $(0 \cdot 021)$, RRM2 (0.008), number of tumour-infiltrating lymphocytes $(-0.009)$, MLPH $(-0.022)$, KRT5 $(-0.024)$ KRT14 (-0.040), MYC (-0.050), PHGDH (-0.050), and BAG1 $(-0 \cdot 168)$.

Two cutoffs based on quartiles were defined to divide patients into low-risk (quartiles 1 and 2), medium-risk (quartile 3), and high-risk (quartile 4) groups. The final model was tested, as a continuous variable and using the prespecified cutoffs, in 267 patients from the evaluation dataset (appendix p 2). The evaluation dataset was composed of patients with gene expression, clinicalpathological, and tumour-infiltrating lymphocytes data from the CHER-LOB and PAMELA studies, and the Padova and Hospital Clinic cohorts. Missing data were not included in our analyses.

Cox proportional hazard regression analyses were used to investigate the association of each variable with survival outcome. Genes associated with the HER2DX risk groups were identified using a multiclass significance analysis of microarrays and a false discovery rate of less than $5 \%$. Categorical variables were expressed as number (\%) and compared between low-risk and medium to high-risk HER2DX groups by $\chi^{2}$ test or Fisher's exact test. Continuous variables were compared by Student's $t$ test. Logistic regression analyses were done to investigate the association of each variable with pathological complete response. The significance level was set to a two-sided $\alpha$ of $0 \cdot 05$. The software used for all statistical analyses was $\mathrm{R}$ version 3.6.2.

\section{Role of the funding source}

The funders of the study had no role in study design, data collection, data analysis, data interpretation, or writing of the report. All authors had full access to all the data in the study and had final responsibility for the decision to submit for publication.

\section{Results}

To build a prognostic model, clinical-pathological and molecular data were available from 435 (35\%) of 1254 patients in the Short-HER trial (table 1). 290 (67\%) patients were placed in the training set and 145 (33\%) in the testing set. Mean age was $55 \cdot 4$ (SD 10.2) and most tumours were $2 \mathrm{~cm}$ or less (T1 stage), node-negative (N0 stage), hormone receptor-positive, histological grade 3 , and had $29 \%$ or less tumour-infiltrating lymphocytes.

Most tumours were PAM50 HER2-enriched and the proportion of HER2-enriched breast cancer was higher in hormone receptor-negative disease (126 patients [70\%]) compared with hormone receptor-positive 


\begin{tabular}{|c|c|c|c|c|}
\hline & $\begin{array}{l}\text { All patients } \\
(n=435)\end{array}$ & $\begin{array}{l}\text { HER2DX } \\
\text { low-risk } \\
(n=218)\end{array}$ & $\begin{array}{l}\text { HER2DX } \\
\text { medium- } \\
\text { risk to } \\
\text { high-risk } \\
(\mathrm{n}=217)\end{array}$ & $p$ value ${ }^{*}$ \\
\hline Age (years) & $55 \cdot 4(10 \cdot 2)$ & $55 \cdot 0(10 \cdot 1)$ & $55 \cdot 7(10 \cdot 4)$ & 0.48 \\
\hline $\begin{array}{l}\text { Tumour- } \\
\text { infiltrating } \\
\text { lymphocytes }\end{array}$ &.. & .. & .. & 0.0001 \\
\hline $0-29 \%$ & $379(87 \%)$ & $176(81 \%)$ & $203(94 \%)$ & .. \\
\hline$\geq 30 \%$ & $56(13 \%)$ & $42(19 \%)$ & $14(7 \%)$ & \\
\hline $\begin{array}{l}\text { Primary tumour } \\
\text { stage }\end{array}$ & & .. & & $<0.0001$ \\
\hline $\mathrm{T} 1$ & $235(54 \%)$ & $157(72 \%)$ & $78(36 \%)$ & .. \\
\hline T2-4 & $200(46 \%)$ & $61(28 \%)$ & $139(64 \%)$ &. \\
\hline Nodal status & .. &.. & .. & $<0.0001$ \\
\hline No & $264(61 \%)$ & $187(86 \%)$ & $77(36 \%)$ & .. \\
\hline N1-3 & $171(39 \%)$ & $31(14 \%)$ & $140(65 \%)$ & .. \\
\hline $\begin{array}{l}\text { PIK3CA } \\
\text { mutations }\end{array}$ &.. & .. & .. & 1.000 \\
\hline Wild type & $339(78 \%)$ & $169(78 \%)$ & $170(78 \%)$ & .. \\
\hline Mutated & $92(21 \%)$ & $46(21 \%)$ & $46(21 \%)$ & .. \\
\hline NA & $4(1 \%)$ & $3(1 \%)$ & $1(1 \%)$ & .. \\
\hline $\begin{array}{l}\text { Hormone } \\
\text { receptor status }\end{array}$ &. & .. & .. & 0.092 \\
\hline Positive & 309 (71\%) & $163(75 \%)$ & $146(67 \%)$ & .. \\
\hline Negative & $126(29 \%)$ & $55(25 \%)$ & $71(33 \%)$ & .. \\
\hline Treatment group & & & .. & 0.63 \\
\hline $\begin{array}{l}\text { 1-year adjuvant } \\
\text { trastuzumab } \\
\text { plus } \\
\text { chemotherapy }\end{array}$ & $222(51 \%)$ & $114(52 \%)$ & $108(50 \%)$ & .. \\
\hline $\begin{array}{l}\text { 9-week } \\
\text { adjuvant } \\
\text { trastuzumab } \\
\text { plus } \\
\text { chemotherapy }\end{array}$ & $213(49 \%)$ & $104(48 \%)$ & $109(50 \%)$ & .. \\
\hline Grade & .. & .. & .. & 0.25 \\
\hline 1 & $6(1 \%)$ & $5(2 \%)$ & $1(1 \%)$ & .. \\
\hline 2 & $115(27 \%)$ & $58(27 \%)$ & $57(27 \%)$ & .. \\
\hline 3 & $309(72 \%)$ & $152(71 \%)$ & $157(73 \%)$ & .. \\
\hline PAM50 & .. & .. & .. & $<0.0001$ \\
\hline Luminal A & $87(20 \%)$ & $63(29 \%)$ & $24(11 \%)$ & .. \\
\hline Luminal B & $43(10 \%)$ & $24(11 \%)$ & $19(9 \%)$ & .. \\
\hline HER2-enriched & $230(53 \%)$ & $75(34 \%)$ & 155 (71\%) & .. \\
\hline Basal-like & $27(6 \%)$ & $17(8 \%)$ & $10(5 \%)$ & .. \\
\hline Normal-like & $48(11 \%)$ & $39(18 \%)$ & $9(4 \%)$ & .. \\
\hline
\end{tabular}

Data are mean (SD) or $\mathrm{n}(\%) . \mathrm{NA}=$ not assessed. *p values represent the comparison between the HER2DX combined prognostic score low-risk group and the medium-risk to high-risk group.

\section{Table 1: Baseline characteristics of the Short-HER patient dataset}

disease (309 [46\%]). As expected, most luminal A or B and basal-like subtypes were hormone receptor-positive (309 [99\%]) and hormone receptor-negative (126 [70\%]), respectively.

A multivariable Cox model analysis of distant metastasis-free survival on the dataset from the 435 patients in Short-HER trial showed that tumour size,

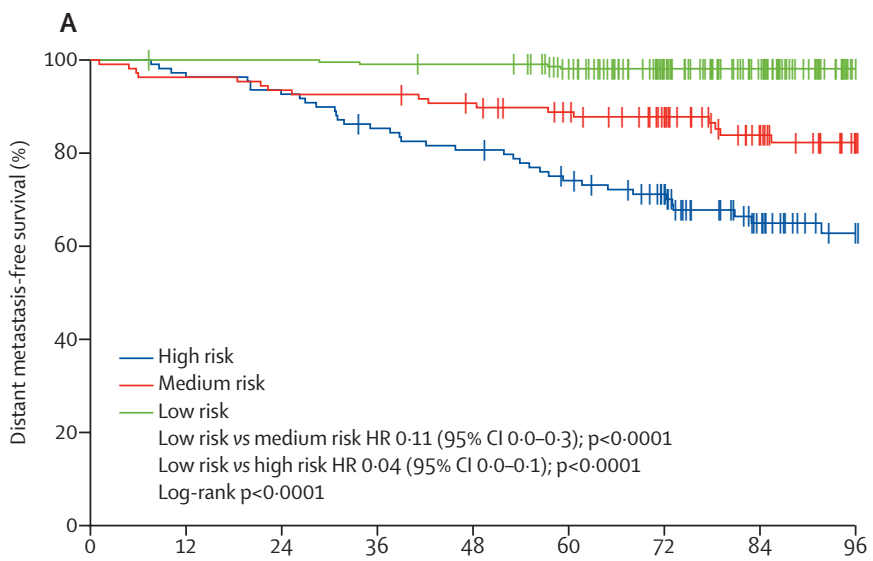

$\begin{gathered}\text { Number at risk } \\ \text { (number censored) }\end{gathered}$
$\begin{array}{rlllllllll}\text { High risk } & 109(0) & 105(0) & 101(0) & 92(1) & 87(1) & 78(3) & 67(11) & 42(31) & 28(44) \\ \text { Medium risk } & 108(0) & 104(0) & 101(0) & 100(0) & 96(2) & 89(7) & 78(17) & 58(34) & 42(49) \\ \text { Low risk } & 218(0) & 217(1) & 217(1) & 215(1) & 214(2) & 203(11) & 173(41) & 137(77) & 92(122)\end{array}$

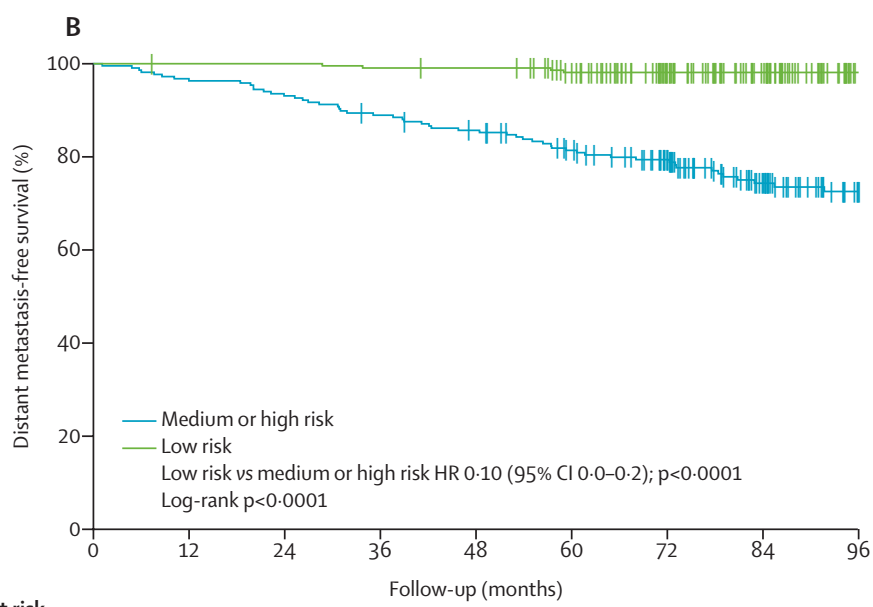

\footnotetext{
Number at risk

(number censored)

Medium or high risk $217(0) \quad 209(0) \quad 202(0) \quad 192(1) \quad 183(3) \quad 167(10) \quad 145(28) \quad 100(65) \quad 70(93)$ Low risk $218(0) \quad 217(1) \quad 217(1) \quad 215(1) \quad 214(2) \quad 203(11) \quad 173(41) \quad 137(77) \quad 92(122)$
}

Figure 1: Distant metastasis-free survival outcomes based on HER2DX combined prognostic scores in the Short-HER training dataset

(A) Distant metastasis-free survival according to low-risk (quartiles 1 and 2 combined), medium-risk (quartile 3) and high-risk (quartile 4) scores. (B) Distant metastasis-free survival according to low-risk (quartiles 1 and 2 combined) and medium or high-risk (quartiles 3 and 4 combined) scores. HR=hazard ratio.

nodal status, percentage of tumour-infiltrating lymphocytes, and PAM50 subtypes were independent prognostic variables (appendix p 3). Next, we evaluated the ability of 31 variables to provide additional prognostic information using cross-validated elastic-net Cox models. The final HER2DX score included 17 variables: tumour size (T1 vs rest), nodal status (N0 vs rest), number of tumourinfiltrating lymphocytes (as a continuous variable), PAM50 subtype (HER2-enriched and basal-like vs rest), and 13 individual genes (appendix pp 13-16). Seven (54\%) of these genes had coefficients associated with poor distant metastasis-free survival outcome and were mostly tracking proliferation-related genes (CDC6, EXO1, and 


\begin{tabular}{|c|c|c|c|c|}
\hline & $\begin{array}{l}\text { All patients } \\
(n=267)\end{array}$ & $\begin{array}{l}\text { HER2DX } \\
\text { low risk } \\
(n=117)\end{array}$ & $\begin{array}{l}\text { HER2DX } \\
\text { medium risk } \\
\text { to high risk } \\
(n=150)\end{array}$ & p value ${ }^{*}$ \\
\hline Age (years) & $54.5(11.8)$ & $53.4(11.8)$ & $55 \cdot 4(11 \cdot 8)$ & 0.48 \\
\hline $\begin{array}{l}\text { Tumour- } \\
\text { infiltrating } \\
\text { lymphocytes }\end{array}$ &.. & .. & .. & 0.0090 \\
\hline $0-29 \%$ & $220(82 \%)$ & $88(75 \%)$ & $132(88 \%)$ & .. \\
\hline$\geq 30 \%$ & $47(18 \%)$ & $29(25 \%)$ & $18(12 \%)$ & .. \\
\hline $\begin{array}{l}\text { Clinical tumour } \\
\text { stage }\end{array}$ &.. &.. & .. & 0.010 \\
\hline $\mathrm{T} 1$ & $57(21 \%)$ & $34(29 \%)$ & $23(15 \%)$ & .. \\
\hline T2-4 & $210(79 \%)$ & $83(71 \%)$ & $127(85 \%)$ & .. \\
\hline $\begin{array}{l}\text { Clinical nodal } \\
\text { status }\end{array}$ &.. & .. & .. & $<0.0001$ \\
\hline No & 148 (55\%) & $101(86 \%)$ & $47(31 \%)$ & .. \\
\hline N1-3 & $119(45 \%)$ & $16(14 \%)$ & $103(69 \%)$ & .. \\
\hline $\begin{array}{l}\text { Pathological } \\
\text { response }\end{array}$ & .. & .. & .. & 0.90 \\
\hline $\begin{array}{l}\text { Complete } \\
\text { response }\end{array}$ & $98(37 \%)$ & $42(36 \%)$ & $56(37 \%)$ & .. \\
\hline $\begin{array}{l}\text { Residual } \\
\text { disease }\end{array}$ & $169(63 \%)$ & $75(64 \%)$ & 94 (63\%) & .. \\
\hline $\begin{array}{l}\text { Hormone } \\
\text { receptor status }\end{array}$ &.. &.. & .. & 0.0001 \\
\hline Positive & $172(64 \%)$ & $91(78 \%)$ & $81(54 \%)$ & .. \\
\hline Negative & $95(36 \%)$ & $26(22 \%)$ & 69 (46\%) & .. \\
\hline Grade &.. & .. & .. & 0.34 \\
\hline 1 & $15(6 \%)$ & $5(5 \%)$ & $10(7 \%)$ & .. \\
\hline 2 & $71(28 \%)$ & $35(32 \%)$ & $36(25 \%)$ &.. \\
\hline 3 & $168(66 \%)$ & $68(63 \%)$ & $100(69 \%)$ & .. \\
\hline PAM50 & .. &.. & .. & $<0.0001$ \\
\hline Luminal A & $51(19 \%)$ & $38(33 \%)$ & $13(9 \%)$ & .. \\
\hline Luminal B & $33(12 \%)$ & $20(17 \%)$ & $13(9 \%)$ & .. \\
\hline $\begin{array}{l}\text { HER2- } \\
\text { enriched }\end{array}$ & $138(52 \%)$ & $35(30 \%)$ & $103(69 \%)$ &.. \\
\hline Basal-like & $21(8 \%)$ & $7(6 \%)$ & $14(9 \%)$ &.. \\
\hline Normal-like & $24(9 \%)$ & $17(15 \%)$ & $7(5 \%)$ & .. \\
\hline Study & .. & .. & .. & 0.37 \\
\hline PAMELA & $88(33 \%)$ & $33(28 \%)$ & $55(37 \%)$ & .. \\
\hline CHER-LOB & $74(28 \%)$ & $38(33 \%)$ & $36(24 \%)$ &.. \\
\hline Hospital Clinic & $68(26 \%)$ & $30(26 \%)$ & $38(25 \%)$ &.. \\
\hline Padova & $37(14 \%)$ & $16(14 \%)$ & $21(14 \%)$ & .. \\
\hline
\end{tabular}

Data are mean (SD) or $\mathrm{n}(\%)$. $\mathrm{p}$ values represent the comparison between the HER2DX combined prognostic score low-risk group and the medium-risk to highrisk groups.

Table 2: Baseline characteristics of the combined patient evaluation dataset

RRM2), HER2-enriched-related biology (TMEM45B and FGFR4) and basal-like-related biology (CDH3). The other six (46\%) genes had survival coefficients associated with better outcome and were mostly tracking luminal A-related biology (BAG1), normal-like biology (KRT5, KRT14, MLPH, and MYC), and basal-like-related biology (PHGDH). The predictive performance (C-index) of HER2DX in Short-HER was 0.80 for all patients,
0.83 for the training set, and 0.72 for the testing set from Short-HER.

HER2DX measured as a continuous variable was significantly associated with distant metastasis-free survival in the Short-HER 435 patient-dataset $(\mathrm{p}<0 \cdot 0001)$. According to HER2DX scoring based on quartiles (appendix p 4), the 5-year distant metastasis-free survival of quartiles 1, 2, 3, and 4 were $97 \cdot 1 \%$ (95\% CI 94·0-100 · ), $99 \cdot 1 \%(97 \cdot 3-100 \cdot 0), 88 \cdot 9 \%(83 \cdot 2-95 \cdot 0)$, and $73.9 \%$ $(66 \cdot 0-82 \cdot 7)$, respectively. There was no significant difference in distant metastasis-free survival between quartile 2 versus quartile 1 (hazard ratio [HR] 0.92, 95\% CI $0 \cdot 23-3 \cdot 70, p=0 \cdot 91)$. Quartiles 3 and 4 had significantly worse distant metastasis-free survival compared with quartile 1 (quartile 3: $\mathrm{HR} 4 \cdot 57,95 \% \mathrm{CI}$ 1.5-13·6, $\mathrm{p}=0 \cdot 010$; quartile 4: $12 \cdot 0,4 \cdot 30-33 \cdot 5$, $\mathrm{p}<0 \cdot 0001)$.

Based on these findings, HER2DX median score (ie, quartiles 1-2) was identified as the cutoff to identify low-risk patients (figure 1). The 5-year distant metastasisfree survival of the low-risk group was $98 \cdot 1 \%$ (95\% CI 96.3-99.9; figure 1). The HER2DX score that distinguished quartile 3 from quartile 4 was designated as the cutoff to identify medium-risk and high-risk patients. 5-year distant metastasis-free survival was 88.9\% (95\% CI 83.2-95.0) in the medium-risk group and $73 \cdot 9 \%(66 \cdot 0-82 \cdot 7)$ in the high-risk group. The low-risk group (quartiles 1-2) had significantly longer distant metastasis-free survival compared with the high-risk group (quartile 4), the medium-risk group (quartile 3), and the medium-risk to high-risk group (quartiles 3-4; figure 1). An analysis of HER2DX versus disease-free survival showed similar results (appendix p 4).

Clinical-pathological and molecular features of the HER2DX low-risk patients in Short-HER were compared with those of the medium-risk to high-risk patients (table 1). No clinical-pathological or molecular feature was unique to HER2DX low-risk patients. Similarly, $7-36 \%$ of HER2DX medium to high-risk patients had features associated with a better survival outcome, such as a high percentage of tumour-infiltrating lymphocytes (>30\%), T1 tumours, or node-negative disease (table 1).

$41(75 \%)$ of 55 genes analysed in total were found differentially expressed across the three risk groups (appendix p 5).

A dataset of 267 patients with early-stage HER2-positive disease obtained from a combined cohort of four neoadjuvant studies was used for an independent evaluation of the HER2DX score (the score was determined at baseline before starting neoadjuvant therapy; table 2). The evaluation dataset was composed of 74 (61\%) of 121 patients from CHER-LOB, 88 (58\%) of 151 from PAMELA, 37 from the Padova cohort and 68 from the Hospital Clinic cohort. All patients received chemotherapy and 1 year of trastuzumab; 116 (43\%) of 267 patients received dual HER2 blockade with lapatinib and trastuzumab for 4.5 to 6.0 months, and $20(8 \%)$ of 267 received four cycles of neoadjuvant pertuzumab. 
Despite heterogeneity in systemic therapies, there were no significant differences in disease-free survival across the four cohorts (appendix p 6).

In the evaluation dataset, HER2DX score as a continuous variable was significantly associated with disease-free survival (HR 2.77, 95\% CI 1.4-5.6, $\mathrm{p}=0.0040$; appendix pp 7,17 ). According to the prespecified cutoffs, the HER2DX low-risk group had longer disease-free survival than the medium-risk to high-risk group or the high-risk group (figure 2). 5-year disease-free survival in the HER2DX low-risk, high-risk, and medium-risk to high-risk groups was $93 \cdot 5 \%$ (95\% CI $89 \cdot 0-98 \cdot 3), 81 \cdot 1 \%(71 \cdot 5-92 \cdot 1)$, and $86 \cdot 7 \%(81 \cdot 2-92 \cdot 5)$, respectively. 8 -year disease-free survival in the HER2DX low-risk, high-risk, and medium to high-risk groups was $91 \cdot 7 \%$ (95\% CI 86.2-97.6\%), 54·1\% (24.1-100), and $78 \cdot 7 \%(62 \cdot 6-98 \cdot 9)$, respectively.

Tumour-infiltrating 1 ymphoc ytes as a continuous variable (odds ratio [OR] 1.04, 95\% CI 1.0-1·1, p<0 0001) and HER2-enriched subtype (OR 3.25, 95\% CI 1·8-5 · , $\mathrm{p}<0 \cdot 0001)$ were associated with pathological complete response in the evaluation cohort. On the contrary, HER2DX score as a continuous variable was not associated with pathological complete response (OR 1.02, 95\% CI 0.6-1.6, $\mathrm{p}=0.93$ ) in the evaluation cohort. According to the previously described cutoffs, the proportion of patients who achieved a pathological complete response in the evaluation cohort in the HER2DX lowrisk, medium-risk, and high-risk groups were 42 (36\%) of 117 patients, 34 (39\%) of 86 patients and 22 (36\%) of 64 patients. Among 169 patients with residual disease in the evaluation cohort, the distribution of HER2DX lowrisk, medium-risk, and high-risk groups was 75 (44\%), $52(31 \%)$, and $42(25 \%)$, respectively. In this setting of patients with residual disease, the HER2DX low-risk group had longer disease-free survival compared with the high-risk group (HR 0 34, 95\% CI $0 \cdot 1-0 \cdot 9, \mathrm{p}=0 \cdot 030$ ) but not the medium-risk group $(0 \cdot 63,0 \cdot 2-1 \cdot 7, \mathrm{p}=0 \cdot 38)$ or the medium-risk to high-risk group $(0 \cdot 47,0 \cdot 2-1 \cdot 1$, $\mathrm{p}=0 \cdot 10$; appendix pp 7-12). In patients with residual disease, 5-year disease-free survival in the HER2DX low-risk and high-risk groups was $90 \cdot 0 \% \quad(95 \% \mathrm{CI}$ $83 \cdot 2-97 \cdot 4)$ and $78 \cdot 2 \%(65 \cdot 6-93 \cdot 2)$, respectivel. 8-year disease-free survival in the HER2DX low-risk and highrisk groups was $87.6 \%$ (95\% CI 79.7-96.3) and 39.1\% $(0 \cdot 1-100 \cdot 0)$, respectively. Of 98 patients who achieved a pathological complete response, the distribution of HER2DX low-risk, medium-risk, and high-risk groups was $42(42 \cdot 9 \%), 34(34 \cdot 7 \%)$, and $22(22 \cdot 4 \%)$, respectively.

\section{Discussion}

To our knowledge, this is the first s tudy a ttempting to build a combined prognostic score based on 17 clinicalpathological and genomic variables in early-stage, HER2positive breast cancer using tumour samples from a phase 3 trial. Specifically, our results showed that HER2DX is associated with long-term distant metastasis-free

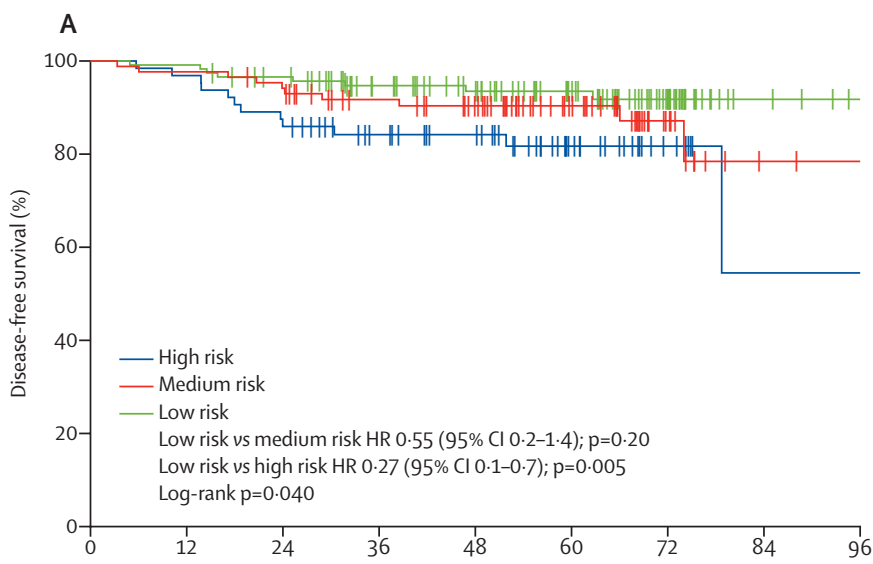

\begin{tabular}{|c|c|c|c|c|c|c|c|c|c|}
\hline $\begin{array}{r}\text { Number at risk } \\
\text { (number censored) }\end{array}$ & & & & & & & & & \\
\hline High risk & $64(0)$ & $62(0)$ & $55(0)$ & $45(9)$ & $39(15)$ & $21(32)$ & $8(45)$ & $2(50)$ & $2(50)$ \\
\hline Medium risk & $86(0)$ & $84(0)$ & 80 (1) & 68 (11) & $60(18)$ & 39 (39) & $14(63)$ & $3(73)$ & $2(74)$ \\
\hline Low risk & $117(0)$ & $116(0)$ & 109 (4) & 89 (22) & $77(33)$ & $58(52)$ & $32(77)$ & $6(103)$ & $2(107)$ \\
\hline
\end{tabular}

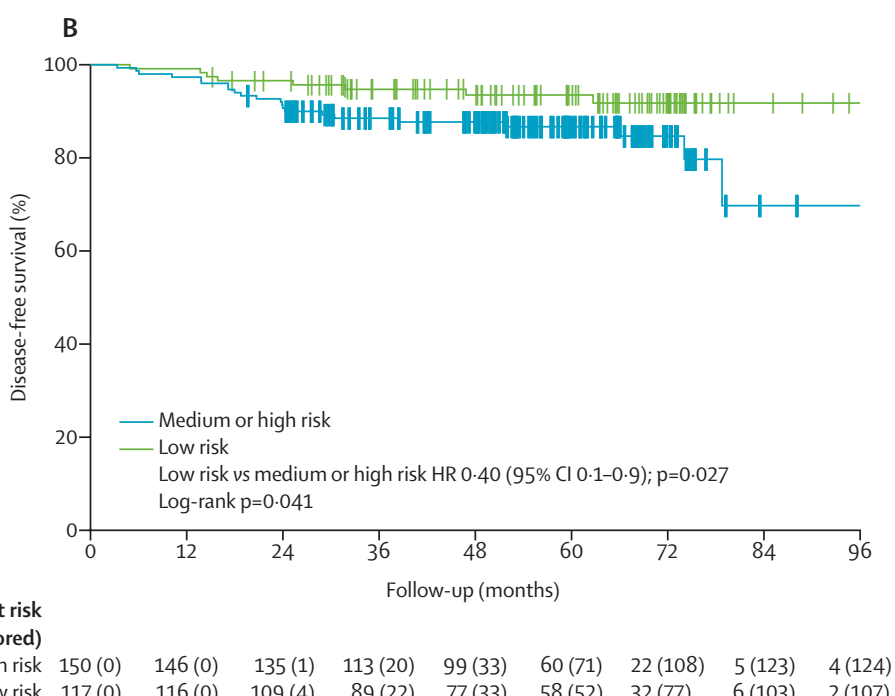

Number at risk (number censored)

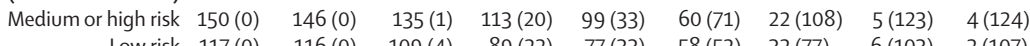
Low risk $117(0) \quad 116(0) \quad 109(4) \quad 89(22) \quad 77(33) \quad 58(52) \quad 32(77) \quad 6(103) \quad 2(107)$

Figure 2: Disease-free survival outcomes based on HER2DX combined prognostic scores in the combined evaluation dataset

(A) Disease-free survival according to low-risk (quartiles 1 and 2 combined), medium-risk (quartile 3) and high-risk (quartile 4) scores. (B) Disease-free survival according to low-risk (quartiles 1 and 2 combined) and medium or high-risk (quartiles 3 and 4 combined) scores. HR=hazard ratio.

survival and can identify groups of patients with different risks of relapsing following standard therapy. Additionally, our study provided insights about the relationship between response to therapy in the neoadjuvant setting and longterm prognosis. From a clinical point of view, HER2DX could identify patients with early-stage, HER2-positive disease who are candidates for escalated or de-escalated systemic treatment. Future validation of HER2DX seems warranted.

Escalation or de-escalation of systemic therapies in early-stage, HER2-positive disease is a controversial topic. In stage 1 disease, the APT trial ${ }^{28}$ showed diseasefree survival rates of $93.3 \%$ following 3 months of 
adjuvant paclitaxel plus 1 year of trastuzumab in a singlearm trial of 410 patients. This treatment strategy is now widely adopted, ${ }^{28}$ although controversy exists in patients with hormone receptor-negative disease. ${ }^{29}$ Regarding de-escalation of trastuzumab, several non-inferiority studies, including the Short-HER trial, ${ }^{22}$ have shown a narrow reduction in recurrence risk with 12 months of therapy compared with shorter durations. ${ }^{10,28,29}$ This treatment strategy, however, has not been widely adopted worldwide, despite its potential impact in low-income countries where trastuzumab is not reimbursed. ${ }^{23}$

In stage 2-3 disease, escalated systemic treatments with pertuzumab, neratinib, and trastuzumab emtansine are approved by the US Food and Drug Administration and the European Medicines Agency. ${ }^{11-13}$ However, the absolute benefit of pertuzumab and neratinib is low $1<3 \%$ in invasive disease-free survival). ${ }^{11,12}$ Trastuzumab emtansine, contrarily, has shown clinically meaningful results with an absolute increase in invasive disease-free survival at 3 years of $11.3 \%$ compared with trastuzumab in patients with HER2-positive breast cancer who do not achieve a pathological complete response following standard anti-HER2-based chemotherapy. ${ }^{13}$ However, three of four patients in the control group of this pivotal tria $1^{13}$ did not have an event at 3 years. Overall, there is an urgent need to better define the populations of patients with early-stage, HER2-positive disease who are candidates for escalated or de-escalated systemic therapies.

To our knowledge, our study is the first to report a clinically valuable prognostic biomarker in HER2-positive breast cancer. Specifically, the HER2DX score can divide the population of early-stage, HER2-positive breast cancer into two prognostically distinct groups. To accomplish this, the assay integrates multiple data types and presents a single prognostic score as a continuous variable and proposes specific cutoffs. Importantly, the HER2DX lowrisk group cannot be identified by classic clinical and pathological variables, and a substantial proportion of HER2DX low-risk patients have individual features known to be associated with poor survival outcome, such as a large tumour size, nodal positivity, a low number of tumour-infiltrating lymphocytes, and residual disease after neoadjuvant therapy. Finally, an intriguing finding is that HER2DX is not associated with the probability to achieve a pathological complete response following antiHER2-based therapy.

Our study had several limitations. First, the evaluation dataset was a heterogeneous cohort of patients. Second, the survival endpoint from the training dataset (ie, distant metastasis-free survival) was different from the evaluation dataset (ie, disease-free survival), because PAMELA had disease-free survival data recorded, not distant metastasisfree survival. Third, the CIs of the survival estimates at 5 years and 8 years of the different risk groups overlap. Fourth, a substantial proportion of patients in the evaluation dataset also received dual HER2 blockade with lapatinib and trastuzumab. However, the absolute effect of dual HER2 blockade with these two drugs in terms of survival outcomes was small (ie, absolute increase compared with trastuzumab of $2 \%$ at 4 years). ${ }^{30}$ Fifth, HER2DX was developed from primary tumour specimens and staging was based on surgical pathology reports. This approach is different from the neoadjuvant setting where a core biopsy is the only available tissue and staging is based on imaging. Despite this limitation, HER2DX did well in the combined neoadjuvant dataset, suggesting it can predict outcome at diagnosis before any treatment is initiated using core biopsies. Sixth, the Short-HER cohort was powered for another primary endpoint, which was to compare disease-free survival between two treatment groups. However, the analysis presented here used all available patients from this study. Thus, we did not do a formal power analysis and focused on significant results. Finally, the HER2DX assay is not standardised and specific cutoffs will need to be defined.

Following our results, the question remains whether HER2DX will guide the use of systemic therapy in earlystage HER2-positive breast cancer. Our opinion is that we are not ready yet to embrace this biomarker and further validation studies should establish its clinical use in different scenarios with a particular focus in the neoadjuvant setting, where the type of pathological response might be incorporated in the HER2DX algorithm. To accomplish this, the HER2DX assay should be standardised and applied retrospectively in tumour samples from at least two large and completed phase 3 pivotal clinical trials such as APHINITY, ${ }^{11}$ NeoALTTO, ${ }^{30}$ ExteNET, ${ }^{12}$ PERSEPHONE, or KATHERINE. ${ }^{13}$ For example, patients with HER2DX low-risk disease at diagnosis who do not achieve a pathological complete response following antiHER2-based neoadjuvant therapy could be spared 14 cycles of adjuvant trastuzumab emtansine. Finally, HER2DX could help the design of prospective clinical trials to test novel escalation or de-escalation treatment strategies.

\section{Contributors}

AP and PC designed the study. AP, PC, LP, GG, and TP contributed to data collection and assembly. AP, PC, LP, and JSP interpreted and analysed the data. All authors wrote and reviewed the report and approved the final version for submission.

\section{Declaration of interests}

AP reports grants and personal fees from Roche, AstraZeneca, Daiichi Sankyo, Merck Sharp \& Dohme (MSD), PUMA Biotechnology, Novartis, and Nanostring Technologies; personal fees from Seattle Genetics, Lilly, Pfizer, Guardant Health, Oncolytics Biotech, and Abbvie, all outside the submitted work and a patent (WO2018/103834A1) licensed to Nanostring Technologies, a patent (WO/2018/096191) issued, and a patent on HER2DX pending. VG reports personal fees from Lilly, Novartis, and Roche; grants from Roche, all outside the submitted work; and a patent on HER2DX pending. LP has a patent on HER2DX pending. AL-C reports grants, personal fees and non-financial support from Novartis, Roche, AstraZeneca, Lilly, and Pfizer; grants and non-financial support from Eisai; grants and personal fees from Genomic Health and GlaxoSmithKline (GSK) Tesaro; personal fees from MSD; and personal fees and non-financial support from Bristol, outside the submitted work. MO reports grants from GSK; grants, personal fees and non-financial support from Roche and Novartis; grants and persona fees from Seattle Genetics, AstraZeneca, and PUMA Biotechnology; 
personal fees from GSK; grants from Genentech, Immunomedics, Boehringer-Ingelheim, Zenith Epigenetics; and non-financial support from Pierre-Fabre, GP Pharma, Grünenthal, and Eisai. AM reports grants and personal fees from Roche and Eisai; personal fees from Lilly, Macrogenics, and Novartis; and grants from Pfizer, all outside the submitted work. RL reports grants from Lilly, Novartis, Pfizer, Merck, Roche, and Bristol-Myers Squibb (BMS); and personal fees from Pharmamar, Bayer, and Pierre Fabre. LM reports personal fees from Roche, AstraZeneca, Novartis, Tesaro, Pfizer, GSK, and Clovis, outside the submitted work. CMP reports personal fees from Bioclassifier LLC outside the submitted work, and a US Patent 12995459 with royalties paid to Veracyte/Nanostring. SP reports personal fees and non-financial support from Novartis; personal fees from Roche; and grants and personal fees from Polyphor, all outside the submitted work. CS reports personal fees from PUMA Biotechnology, Pfizer, F Hoffmann-La Roche, AstraZeneca, Celgene, Daiichi Sankyo, Genomic health, Novartis, Pierre Fabre, Synthon Biopharmaceuticals, MSD, Odonate Therapeutics, Philips Healthwork, prIME Oncology, and Sanofi Aventis; and grants from Roche-Genentech, Macrogenics, Pfizer, Piqur Therapeutics, PUMA Biotechnology, Synthon Biopharmaceuticals, Novartis, and BMS, all outside the submitted work. JSP has a patent with royalties paid to Nanostring. JC reports grants and personal fees from Roche, AstraZeneca, Daiichi Sankyo, and MSD; personal fees from Celgene, Cellestia, Biothera Pharmaceutical, Merus, Seattle Genetics, Erytech, Athenex, Polyphor, Lilly, Servier, GSK, Leuko, Bioasis, Clovis Oncology, and Boehringer Ingelheim; grants from Pfizer, Bayer, Eisai, Guardant Health, PUMA Biotechnology, Ariad Pharmaceuticals, and Baxalta $\mathrm{GmbH}$, all outside the submitted work; and has a patent (WO2018/103834A1) licensed to Nanostring Technologies. MV reports personal honoraria from Pfizer, Novartis, Roche, and Daiichi Sankyo; travel, accommodation, and expenses paid by Roche and Pfizer; and a paid consulting or advisory role for Roche and Novartis. EC reports personal fees from Pfizer, Roche, Novartis, and Lilly, during the conduct of this study. MVD reports personal fees from Lilly, Genomic Health, and Celgene, outside the submitted work; and has a patent HER2DX pending. AF reports personal fees from Roche, Novartis, and Pfizer, outside the submitted work. PV reports personal fees from Nanostring Technologies, outside the submitted work. PC reports grants from Merck; grants and personal fees from Roche; personal fees from Novartis and Lilly, all outside the submitted work; and has a patent on HER2DX pending. All remaining authors declare no competing interests.

\section{Data sharing}

The data collected for the study will not be made publicly available. We encourage investigators interested in data sharing and collaboration to contact the corresponding author (AP).

\section{Acknowledgments}

This study was funded by Instituto de Salud Carlos III, Save the Mama, Pas a Pas, Fundación Científica Asociación Española Contra el Cáncer, Fundación SEOM, Agenzia Italiana del Farmaco, Italian Association for Cancer Research, the Veneto Institute of Oncology, National Institutes of Health (R01CA229409), and International Agency for Research on Cancer.

\section{References}

1 Perez EA, Romond EH, Suman VJ, et al. Trastuzumab plus adjuvant chemotherapy for human epidermal growth factor receptor 2-positive breast cancer: planned joint analysis of overall survival from NSABP B-31 and NCCTG N9831. J Clin Oncol 2014; 32: $3744-52$

2 Prat A, Carey LA, Adamo B, et al. Molecular features and survival outcomes of the intrinsic subtypes within HER2-positive breast cancer. J Natl Cancer Inst 2014; 106: dju152.

3 Ferrari A, Vincent-Salomon A, Pivot X, et al. A whole-genome sequence and transcriptome perspective on HER2-positive breast cancers. Nat Commun 2016; 7: 12222.

4 Prat A, Pascual T, De Angelis C, et al. HER2-enriched subtype and ERBB2 expression in HER2-positive breast cancer treated with dual HER2 blockade. J Natl Cancer Inst 2019; 112: 46-54.

5 Brasó-Maristany F, Griguolo G, Pascual T, et al. Phenotypic changes of HER2-positive breast cancer during and after dual HER2 blockade. Nat Commun 2020; 11: 385.
6 Brandão M, Caparica R, Malorni L, Prat A, Carey LA, Piccart M. What is the real impact of estrogen receptor status on the prognosis and treatment of HER2-positive early breast cancer? Clin Cancer Res 2020; 26: 2783-88.

7 Hayes DF. HER2 and breast cancer-a phenomenal success story. N Engl J Med 2019; 381: 1284-86.

8 Veeraraghavan J, De Angelis C, Reis-Filho JS, et al. De-escalation of treatment in HER2-positive breast cancer: determinants of response and mechanisms of resistance. Breast 2017; 34: S19-26.

9 Tolaney SM, Barry WT, Dang CT, et al. Adjuvant paclitaxel and trastuzumab for node-negative, HER2-positive breast cancer. N Engl J Med 2015; 372: 134-41.

10 Pivot X, Romieu G, Debled M, et al. 6 months versus 12 months of adjuvant trastuzumab in early breast cancer (PHARE): final analysis of a multicentre, open-label, phase 3 randomised trial. Lancet 2019; 393: 2591-98.

11 von Minckwitz G, Procter M, de Azambuja E, et al. Adjuvant pertuzumab and trastuzumab in early HER2-positive breast cancer. N Engl J Med 2017; 377: 122-31.

12 Martin M, Holmes FA, Ejlertsen B, et al. Neratinib after trastuzumab-based adjuvant therapy in HER2-positive breast cancer (ExteNET): 5-year analysis of a randomised, double-blind, placebocontrolled, phase 3 trial. Lancet Oncol 2017; 18: 1688-700.

13 von Minckwitz G, Huang C-S, Mano MS, et al. Trastuzumab emtansine for residual invasive HER2-positive breast cancer. N Engl J Med 2018; 380: 617-28.

14 Conte P, Griguolo G, Dieci MV, et al. PAM50 HER2-enriched subtype as an independent prognostic factor in early-stage HER2+ breast cancer following adjuvant chemotherapy plus trastuzumab in the ShortHER trial. Proc Am Soc Clin Oncol 2019; 37 (suppl 15): 544

15 Sparano JA, Gray RJ, Makower DF, et al. Adjuvant chemotherapy guided by a 21-gene expression assay in breast cancer. $N$ Engl J Med 2018; 379: 111-21

16 Parker JS, Mullins M, Cheang MCU, et al. Supervised risk predictor of breast cancer based on intrinsic subtypes. J Clin Oncol 2009; 27: 1160-67.

17 Salgado R, Denkert C, Campbell C, et al. Tumor-infiltrating lymphocytes and associations with pathological complete response and event-free survival in HER2-positive early-stage breast cancer treated with lapatinib and trastuzumab: a secondary analysis of the NeoALTTO trial. JAMA Oncol 2015; 1: 448-54.

18 Krop IE, Paulson J, Campbell C, et al. Genomic correlates of response to adjuvant trastuzumab $(\mathrm{H})$ and pertuzumab $(\mathrm{P})$ in HER2+ breast cancer (BC): biomarker analysis of the APHINITY trial. Proc Am Soc Clin Oncol 2019; 37(suppl 15): 1012.

19 Schettini F, Pascual T, Conte B, et al. HER2-enriched subtype and pathological complete response in HER2-positive breast cancer: a systematic review and meta-analysis. Cancer Treat Rev 2020; 84: 101965

20 Loibl S, Majewski I, Guarneri V, et al. PIK3CA mutations are associated with reduced pathological complete response rates in primary HER2-positive breast cancer: pooled analysis of 967 patients from five prospective trials investigating lapatinib and trastuzumab. Ann Oncol 2016; 27: 1519-25.

21 Llombart-Cussac A, Cortés J, Paré L, et al. HER2-enriched subtype as a predictor of pathological complete response following trastuzumab and lapatinib without chemotherapy in early-stage HER2-positive breast cancer (PAMELA): an open-label, singlegroup, multicentre, phase 2 trial. Lancet Oncol 2017; 18: 545-54.

22 Cortazar P, Zhang L, Untch M, et al. Pathological complete response and long-term clinical benefit in breast cancer: the CTNeoBC pooled analysis. Lancet 2014; 384: 164-72.

23 Pondé N, Gelber RD, Piccart M. PERSEPHONE: are we ready to de-escalate adjuvant trastuzumab for HER2-positive breast cancer? NPJ Breast Cancer 2019; 5: 1.

24 Conte P, Frassoldati A, Bisagni G, et al. Final analysis of the phase III multicentric Italian study Short-HER: 9 weeks vs 1 year adjuvant trastuzumab for HER2+ early breast cancer. Ann Oncol 2018; 29: 2328-33.

25 Guarneri V, Frassoldati A, Bottini A, et al. Preoperative chemotherapy plus trastuzumab, lapatinib, or both in human epidermal growth factor receptor 2-positive operable breast cancer: results of the randomized phase II CHER-LOB study. J Clin Oncol 2012; 30: 1989-95. 
26 Dieci MV, Prat A, Tagliafico E, et al. Integrated evaluation of PAM50 subtypes and immune modulation of pCR in HER2-positive breast cancer patients treated with chemotherapy and HER2-targeted agents in the CherLOB trial. Ann Oncol 2016; 27: 1867-73.

27 Salgado R, Denkert C, Demaria S, et al. The evaluation of tumorinfiltrating lymphocytes (TILs) in breast cancer: recommendations by an international TILs Working Group 2014. Ann Oncol 2015 26: $259-71$

28 Tolaney SM, Guo H, Pernas S, et al. Seven-year follow-up analysis of adjuvant paclitaxel and trastuzumab trial for node-negative, human epidermal growth factor receptor 2-positive breast cancer. J Clin Oncol 2019; 37: 1868-75.
29 Joensuu H, Fraser J, Wildiers H, et al. Effect of adjuvant trastuzumab for a duration of 9 weeks vs 1 year with concomitant chemotherapy for early human epidermal growth factor receptor 2-positive breast cancer: the SOLD randomized clinical trial. JAMA Oncol 2018; 4: 1199-206.

30 Piccart-Gebhart M, Holmes E, Baselga J, et al. Adjuvant lapatinib and trastuzumab for early human epidermal growth factor receptor 2-positive breast cancer: results from the randomized phase III adjuvant lapatinib and/or trastuzumab treatment optimization trial. J Clin Oncol 2016; 34: 1034-42. 